\title{
Essentials of the macroscopic-microscopic folded-Yukawa approach and examples of its record in providing nuclear-structure data for simulations
}

\author{
Peter Möller ${ }^{1, \star}$ \\ ${ }^{1}$ P. Moller Scientific Computing and Graphics, Inc., P. O. Box 1440, Los Alamos, NM 87544, USA
}

\begin{abstract}
The macroscopic-microscopic model based on the folded-Yukawa singleparticle potential and a "finite-range" macroscopic model is probably the approach that has provided the most reliable predictions of a large number of nuclear-structure properties for all nuclei between the proton and neutron drip lines. I will describe some basic features of the model and the development philosophy that may be the reason for its success. Examples of quantities modeled within the same model framework are, nuclear masses, ground-state level structure, including spins, ground-state shapes, fission barriers, heavy-ion fusion barriers, sub-barrier fusion cross sections, $\beta$-decay half-lives and delayed neutron emission probabilities, shape coexistence, and $\alpha$-decay $Q_{\alpha}$ energies to name a few. I will show how well it predicted various properties measured after published results. Rather than giving an incomplete model description here I will give a timeline of model development and provide references to typical applications and references that are sufficiently complete that several individuals have written computer codes based on these references, codes whose results have excellent agreement with ours.
\end{abstract}

\section{Introduction}

Most insight into and understanding of nuclear properties have historically and are currently obtained in terms of surprisingly simple models. In systems which depend mainly on electron behavior their properties can be obtained from solving the Schödinger equation with realistic, known Coulomb potentials. For the nuclear system with its up to a few hundred nucleons a many-body a Scrödinger equation with a potential based on the much more complicated nuclear forces cannot be solved in practice. Therefore other types of models are used. They are often referred to in a condescending manner as phenomenological, but, in a sense all "models" are phenomenological. We will here focus on the remarkable insights into nuclear properties that have been obtained by the "liquid-drop model", the single-particle model and a combination of those, the "macroscopic-microscopic" method that still today is providing much insight about the properties of nuclei.

\section{The liquid-drop model}

The liquid-drop model has its origin in the semi-empirical mass model, usually attributed to von Weizäcker [1] and Bethe and Bacher [2]. In the liquid drop model the nuclear binding energy, which

^e-mail: mollerinla@gmail.com 


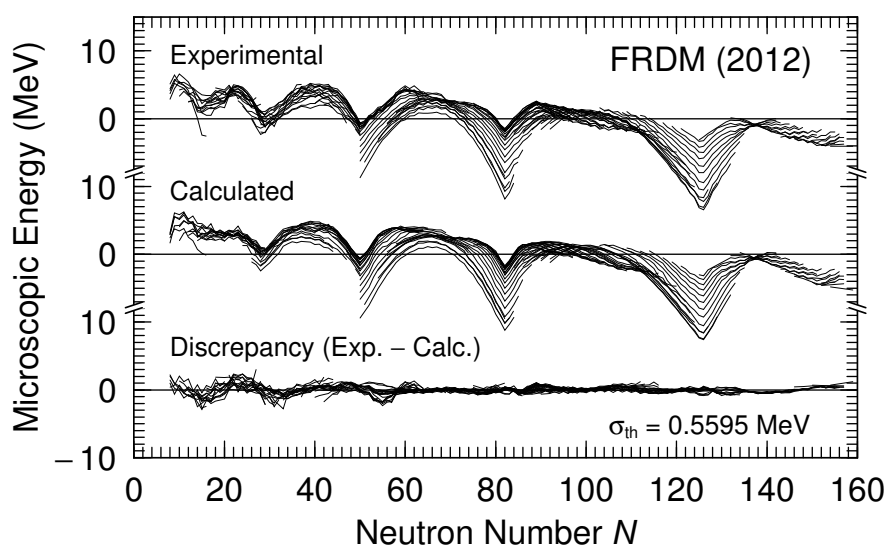

Figure 1. Comparison of experimental and calculated microscopic energies $E_{\text {mic }}$ for 2149 nuclei, for a macroscopic model corresponding to the FRDM. The bottom part showing the difference between these two quantities is equivalent to the difference between measured and calculated ground-state masses. There are almost no systematic errors remaining for nuclei with $N \leq 65$, for which region the error is only $0.355 \mathrm{MeV}$. The results shown in this figure represent our new mass model. The lines are drawn through isotope chains. From Ref. [6].

governs nuclear stability with respect to $\beta$ and $\alpha$ decay, is the sum of a volume energy term, due to short-range "nuclear" forces, a Coulomb energy term due to the repulsive force between protons, a surface tension term, a symmetry energy term with its origin in the Pauli principle, and finally a pairing term.

$$
B(N, Z)=+a_{\mathrm{v}} A-a_{\mathrm{s}} A^{2 / 3}-a_{\mathrm{C}} \frac{Z^{2}}{A^{1 / 3}}-a_{I} \frac{(N-Z)^{2}}{A}-\delta(A)
$$

This simple expression gives the nuclear binding energy, which for a heavy nucleus is roughly $1600 \mathrm{MeV}$ (8 MeV per nucleon) to an accuracy of one percent. The terms represent in order volume energy, surface energy, Coulomb energy, symmetry energy and a pairing correction. The notation $Z, N$, and $A$ stand for proton number, neutron number and total nucleon number, respectively. It was highly useful in interpreting the decay chains following element transmutations. However, in some experiments in which uranium was bombarded with neutrons a confusing number of radioactive decay periods, which were difficult to reconcile with properties given by the semi-empirical mass model, were observed. This was all explained when Hahn and Strassmann [3] identified barium in the products following neutron irradiation of uranium and Meitner and Frisch [4] suggested one could think of the nucleus as a deformable charged liquid drop with a surface tension that had split into two smaller nuclei of about equal size in fission. Arguments based on this simple model also showed that the kinetic energies of the two fragments would be very large and Frisch within weeks of the Hahn and Strassmann experiment submitted a paper to Nature [5] in which his measurements confirmed the expected high kinetic energies, thus giving independent confirmation of the fission process. It then turned out that the semi-empirical mass model could be applied to describe this division and Bohr and Wheeler just a few months later suggested the following generalization [7]

$$
B(N, Z)=+a_{\mathrm{v}} A-a_{\mathrm{s}} A^{2 / 3} B_{\mathrm{s}}(\alpha)-a_{\mathrm{C}} \frac{Z^{2}}{A^{1 / 3}} B_{\mathrm{C}}(\alpha)-a_{I} \frac{(N-Z)^{2}}{A}-\delta(A)
$$

Only the Coulomb and surface energies depend on deformation. $B_{\mathrm{s}}(\alpha)$ and $B_{\mathrm{C}}(\alpha)$ are the ratios of the surface and Coulomb energies at deformation $\alpha$ to that for spherical shape. With this simple model one can show that stability with respect to fission decreases with increasing proton number and is completely lost at proton numbers somewhat above $Z=100$. 
New Masses in AME2012 Relative to AME1989,

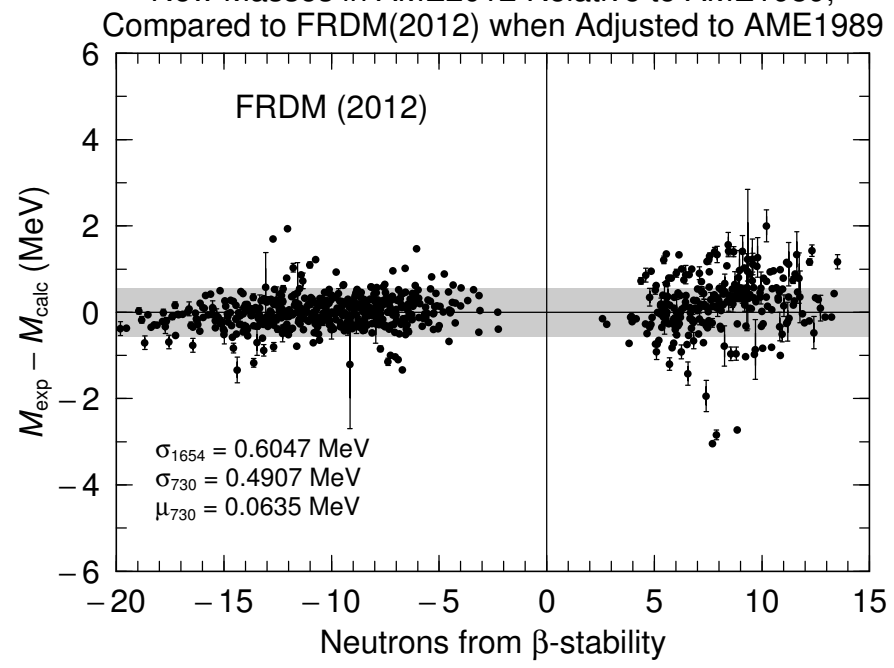

Figure 2. We have adjusted our current mass model (with all the improvements discussed in [6]) to the older AME1989 experimental evaluation to test the extrapability of the model. It agrees better with the AME1989 data base than FRDM(1992), due to improvements in the calculations, $0.6047 \mathrm{MeV}$ versus $0.669 \mathrm{MeV}$ for the previous $\operatorname{FRDM}(1992)$. But it also extrapolates much better with only $0.4948 \mathrm{MeV}$ deviations for the new nuclei, versus 0.5817 for the previous FRDM(1992)[8]. From Ref. [6].

\section{Single-particle model}

Already Bethe and Bacher [2] considered the possibility that microscopic effects might give rise to deviations from the semi-empirical mass model. They noted that in an oscillator single-particle potential there are large gaps in the corresponding single-particle level spectrum and investigated if there were unusually large deviations between their theory and measured masses at $Z=20, N=20$, namely ${ }^{40} \mathrm{Ca}$. They found none, but concluded that the masses were not measured sufficiently accurately to observe the effect they expected. In Fig. 1 we compare in the top panel masses calculated in a modern liquid-drop model [6] to measured masses [9]. We observe that there are no particularly large deviations at these nucleon numbers. But for heavier nuclei there are large deviations at the by now well-known magic numbers. Around 1950 a modern single-particle model was developed [10-12]. It showed large gaps at the nucleon numbers where there were large deviations between the semiempirical mass model and measured masses and it also agreed with observed ground-state spins near magic numbers. But it soon because clear that for large regions of the nuclear chart ground-state spins were not well explained. This observations together with the experimental observations that nuclei in these regions had large intrinsic quadrupole moments led to the suggestion that these nuclei were not spherical but deformed. Motivated by these developments Nilsson in 1955 generalized the spherical single-particle model to describe levels in deformed nuclei for any prescribed spheroidal shape. A large part of the work was to devise a model specification that could be implemented on computers of the day. Nilsson solved this by introducing coordinate transformations that resulted in matrices of low dimensionality about 10 or so [13]. The model results for ground-state spins and low-lying level structure were shown to agree well with experimental observations [14] over a large region of the nuclear chart. It has been one aim of PM to follow this "Copenhagen-Lund" tradition and select viable models based on their ability to describe nuclear properties across the nuclear chart, and also introduce the aim of universality, that is that many nuclear properties are described within a consistent model framework. Before we list our timeline of studies to provide nuclear properties of useful accuracy for astrophysical modeling we need to introduce the third huge development leading to the macroscopic-microscopic method, the Strutinsky shell correction method. 


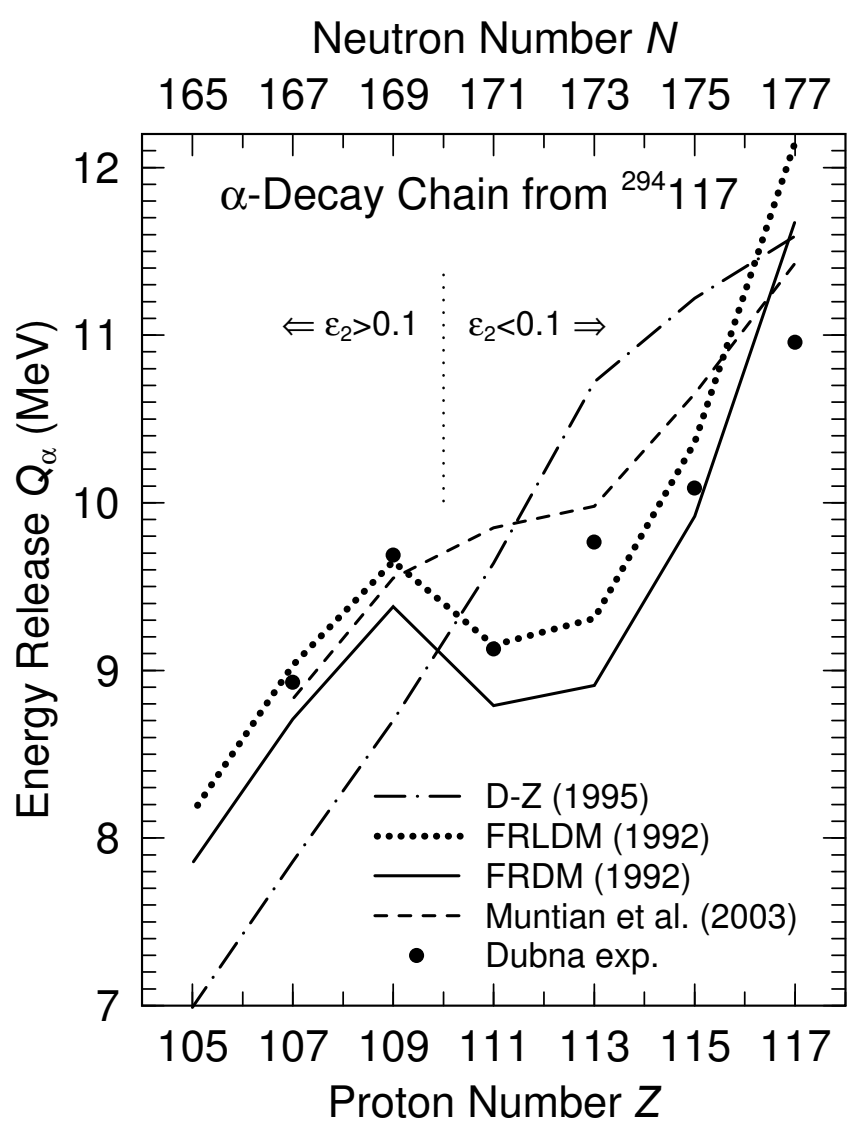

Figure 3. Calculated and measured $Q_{\alpha}$-decay energies for ${ }^{294} \mathrm{Ts}$. The calculated values assume ground-state to ground-state decay, the measured energies may not be, so the differences between measured and calculated values do not directly indicate the degree of (in)accuracy of the underlying mass model. Note that in two of the models the kink observed in the experimental data at $Z=107-109$ is well reproduced. In this case the kink is related to a change in deformation from spherical to deformed, rather than a gap in the level spectrum at a fixed deformation. D-Z is from Ref. [17] and Muntian from Ref. [18].

\section{Strutinsky shell-correction method}

Strutinsky introduced a method that combined physics from the macroscopic liquid-drop model and the single-particle model $[15,16]$. Briefly, the potential energy for any prescribed deformation is calculated in the following manner. The energy for the charged liquid drop is calculated for this shape, somewhat simplified it is the sum of a surface and electrostatic Coulomb energy. The singleparticle energies are calculated. A shell-plus-pairing correction is then obtained from the calculated single-particle spectrum. In Strutinsky's method the correction is about $-6 \mathrm{MeV}$ when there is a large gap in the spectrum such as in the neutron spectrum at $N=126$ for ${ }^{208} \mathrm{~Pb}$. Since there is also a gap in the proton levels at $Z=82$ the total shell+plus-pairing correction at spherical shape is about $-12 \mathrm{MeV}$. In situations where there is a high density of levels the shell-plus-pairing correction sum is larger than $10 \mathrm{MeV}$. We now give some examples of how we have used the macroscopic-microscopic method to provide a multitude of nuclear properties across the nuclear chart. With the continuing increase of computer power over decades it is only during the past 20 or so years this method could be exploited to its full potential. 


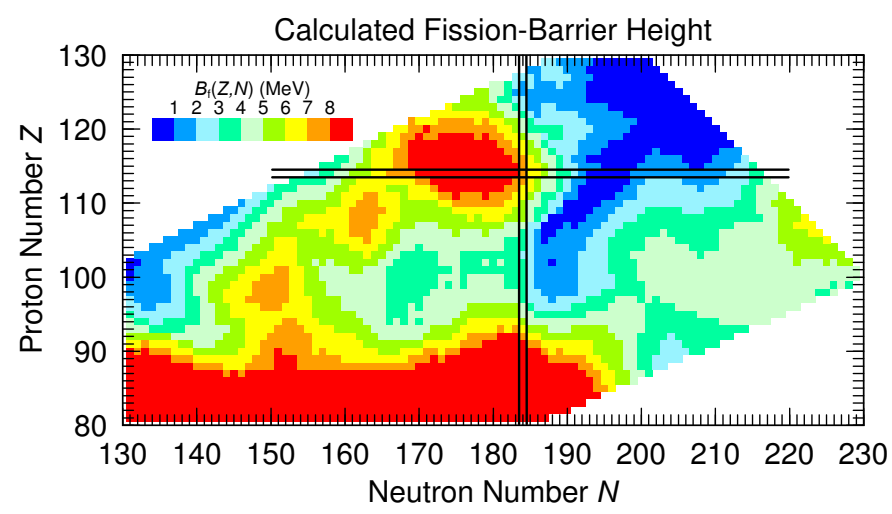

Figure 4. Calculated fission-barrier heights for 3282 nuclei. The highly variable structure is mostly due to ground-state shell effects. Ground-state shell effects are particularly strong in the deformed regions around ${ }_{100}^{252} \mathrm{Fm}_{152},{ }_{108}^{270} \mathrm{Hs}_{162}$ and in the nearly spherical region near the next doubly magic nuclide postulated to be at ${ }_{114}^{298} \mathrm{Fl}_{184}$. The strongest shell effects in this calculation are slightly offset to the left with respect to this isotope. From Ref. [27].

\section{Applications of the macroscopic-microscopic method}

The first development in this sequence was the introduction of the folded-Yukawa single-particle potential in 1972 [19]. The treatment of the spin-orbit force contribution to the potential has a decisive impact on its success. In the discovery paper the authors used a spin-orbit strength determined in Ref. [20]. In 1973 PM and S-G Nilsson did an extensive study of the optimum spin-orbit strength in the folded-Yukawa and decided on values that are still used today [21]. The first macroscopicmicroscopic mass model based on these ideas and an improved macroscopic finite-range "liquid-drop" model [22] appeared in 1981 [23, 24]. Unfortunately, in hindsight it only included about 4000 nuclei, not sufficient to include all r-process nuclei or all nuclei of future interest in the superheavy region. The argument at the time was that we should not blindly let the code crank out numbers without further test of the reliability of the theory. Once comparisons with various types of experimental data and increased computer power were available a mass table extending from the proton to the neutron drip line was completed [8]. This investigation provided masses based on two different macroscopic models, the finite-range liquid-drop model and the finite-range droplet model. It turned out that the latter significantly improved mass predictions. We also note that the expressions used for the neutron and proton single-particle potential radius and depths were since 1972 [19] based on the droplet model ideas and subsequently found to be very realistic and essential [6]. Further development and improved computer performance led to the current mass model FRDM(2012) [6]. As several other groups have done by now, implementation of the FRDM/FRLDM macroscopic-microscopic model can be done based on the complete specifications and mathematical detail found in Refs $[6,8,19,22,25,26]$. It might be useful to note that in Ref. [19] some misprints in Ref. [25] are enumerated.

With this model we also obtain ground-state multipole moments, or equivalently nuclear groundstate shapes. Special types of rare shapes are axially asymmetric and reflection asymmetric shapes. Calculated occurrences of this type of shapes are discussed in Refs. [28, 29].

For some nuclei the potential-energy function versus shape can have several minima. One example is the fission isomeric states which minima correspond to a nuclear shape elongation with the major and minor axes having a ratio of 2:1. Another situation is when there are two minima: one oblate and one prolate, but none as deformed as the fission isomeric state. This situation is referred to as shape coexistence. We have studied globally shape coexistence in Refs. [31, 32].

However, of most interest is perhaps not how well models agree with known data to which its fitted parameters were adjusted. Very important is how reliably properties can be predicted. In Refs. $[33,34]$ it was shown that the differences between the previously published masses [8] and several hundred new measurements were substantially smaller than in the region to which the model param- 


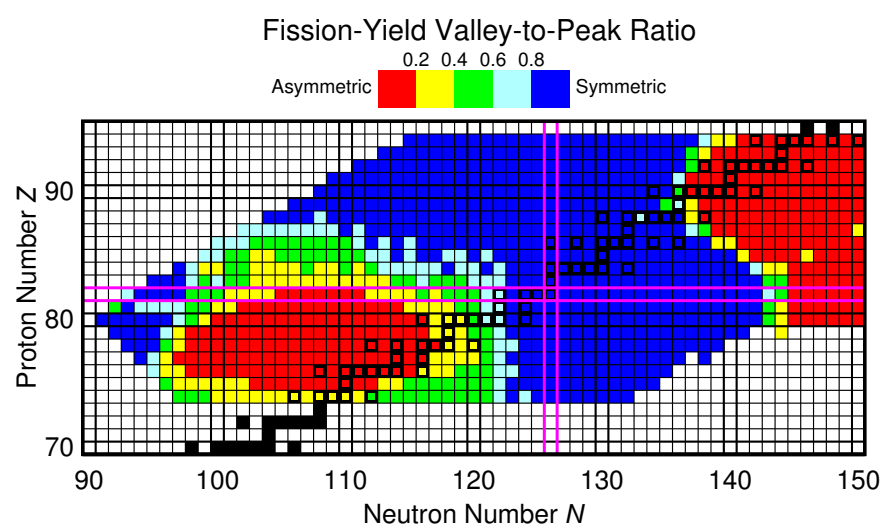

Figure 5. Calculated symmetric-yield to peak-yield ratios for 987 fissioning systems. Black squares (open in colored regions, filled outside) indicate $\beta$-stable nuclei. We find a new, contiguous region of asymmetric fission separated from the classical location of asymmetric fission in the actinides by an extended area of symmetric fission. From Ref. [30].

eters were adjusted. To obtain similar insight we have adjusted the parameters of the FRDM(2012) to a more limited mass data set [35] and studied the calculated masses obtained for masses measured subsequently. The agreement with subsequently measured masses is shown in Fig. 2 and shows encouraging predictive capability. Another way to assess the predictive properties is to compare do superheavy element (SHE) properties. Some of their properties are obtained through their $\alpha$-decay chains. In Fig. 3 we show a comparison of the FRDM(1992) predictions to more recently observed [36] $\alpha$-decay $Q$ values of the decay of ${ }^{294}$ Ts. It is clear the predictions well reproduce the observed interesting structure in the decay chain.

In fission, as we remarked above, the nucleus evolves from a single (ground-state) shape to two separated fission fragments. This process can be studied in the macroscopic-microscopic model by studying the energy of nuclear shapes involved in the transition between the ground state and separated fragments. A study of a few selected shapes (the only possibility at that time with the limited computer power) showed a definite signature of asymmetric fission for actinides but symmetric fission for somewhat lighter nuclei $[37,38]$. It was much later that the macroscopic-microscopic method could be exploited by calculating the potential energy between the ground state and separated fragments for the selection of shapes that should be considered, namely elongation, neck radius, left and right fragment spheroidal deformation, and mass split between the fragments [39]. A poorly appreciated aspect in this type of calculations is: what is required to identify correctly fission saddle points? A more in-depth account of these aspects is in Ref. [40]. Calculated fission barrier-heights based on these methods are in Ref. [27]. We show in Fig. 43282 barrier heights calculated in this approach.

For many simulation applications it is of interest to have available the fission-fragment mass distributions. Recently we showed that a Brownian shape motion on calculated five-dimensional potentialenergy surfaces well reproduced the observed fission-fragment mass distributions [41-43].

Encouraged by these studies which showed very good agreement with experimental fission fragment mass distributions, we went ahead, as we usually do, and made some predictions, so that our model could be tested, of fission fragment mass distributions for an extended region of 987 heavy nuclei [30]. We found a new contiguous region of nuclei predicted to undergo asymmetric fission, see Fig. 5. An earlier "discovery" experiment [44] (which motivated our study) had shown that ${ }^{180} \mathrm{Hg}$ fissioning asymmetrically in $\beta$-delayed fission. There are still only scattered experiments of fragment distributions in this region, mainly in the transition regions between symmetric and asymmetric fission, so the predictions still await serious experimental studies. Currently in progress are calculations of fission yields of r-process nuclei for use in r-process fission recycling modeling. 


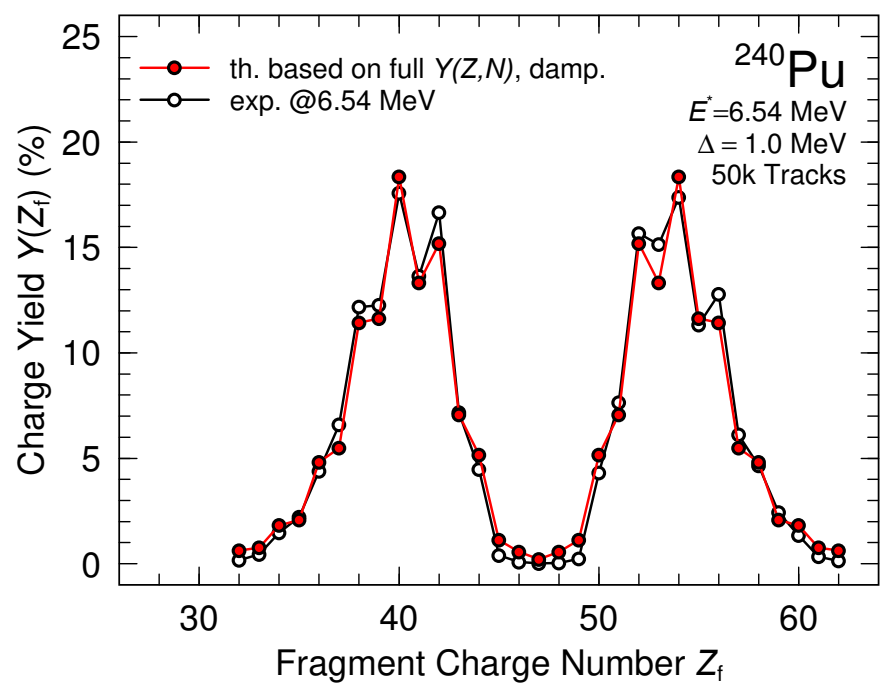

Figure 6. Calculated fission-fragment charge yields in thermal neutron-induced fission of ${ }^{239} \mathrm{Pu}$. The calculated yield is based on a calculation of $Y(Z, N)$ in a recent extension of the Brownian shape-motion model [45] which is then summed over neutron number.

Recently we have generalized the Brownian shape-motion model so that we now model the fissionfragment yields $Y(Z, N)$. The modification is not so much in the Brownian shape-motion model but rather in devising a method to calculate the potential energy of the fissioning nucleus for different $Z / N$ ratios in the two emerging fragments. This leads to two shape asymmetry variables one in $Z$ and one in $N$. The method and benchmarks are presented in Ref. [45]. In Fig. 6 we compare calculated and experimental charge distributions of ${ }^{240} \mathrm{Pu}$. To obtain the charge distribution we summed for each $Z$ the calculated $Y(Z, N)$ over neutron number $N$.

Another substantial modeling effort has been and is to calculate $\beta$-decay properties, specifically decay half-lives and delayed neutron-emission probabilities. Our first global results are in Ref. [33]. A next generation, improved calculation and release will be available in the first half of 2018 . PM maintains a web page with publications, figures appearing in these publications and interactive features at URL: http://t2.lanl.gov/nis/molleretal/.

\section{References}

[1] C. F. von Weizäcker, Z. Phys. 96 (1935) 431

[2] H. A. Bethe and R. F. Bacher, Rev. Mod. Phys. 8 (1936) 82

[3] O. Hahn and F. Strassmann, Naturwiss. 27 (1939) 11

[4] L. Meitner and O. R. Frisch, Nature 143 (1939) 239

[5] O. R. Frisch, Nature 143 (1939) 276

[6] P. Möller, A. J. Sierk, T. Ichikawa, and H. Sagawa, Aтomic Data and Nuclear Data Tables 109-110 (2016) 1

[7] N. Bohr and J. A. Wheeler, Phys. Rev. 56 (1939) 426

[8] P. Möller, J. R. Nix, W. D. Myers, and W. J. Swiatecki, Атомic Data Nucl. Data Tables 59 (1995) 185

[9] M. Wang, G. Audi, A. H. Wapstra, F. G. Kondev, M. MacCormick, X. Xu, and B. Pfeiffer, Chin. Phys. C36 (2012) 1603

[10] M. Mayer, Phys. Rev. 75 (1949) 1969

[11] M. Mayer, Phys. Rev. 78 (1950) 16 
[12] M. Mayer, Phys. Rev. 78 (1949) 22

[13] S. G. Nilsson, Kgl. Danske Videnskab. Selskab. Mat.-Fys. Medd. 29:No. 16 (1955)

[14] B. R. Mottelson and S. G. Nilsson, Kgl. Danske Videnskab. Selskab. Mat.-Fys. Skr. . 1:No. 8 (1959)

[15] V. M. Strutinsky, Nucl. Phys. A95 (1967) 420

[16] V. M. Strutinsky, Nucl. Phys. A122 (1968) 1

[17] J. Duflo and A. P. Zuker, Phys. Rev. C 52 (1995) R23

[18] I. Muntian, Z. Patyk, and A. Sobiczewski, Physics of Atomic Nuclei, 66 (2003) 1015

[19] M. Bolsterli, E. O. Fiset, J. R. Nix, and J. L. Norton, Phys. Rev. C 5 (1972) 1050

[20] W. Ogle, S. Wahlborn, R. Piepenbring, and S. Fredriksson, Rev. Mod. Phys. 43 (1971) 424

[21] P. Möller and J. R. Nix, Proc. Third IAEA Symp. on the physics and chemistry of fission, Rochester, 1973, vol. I (IAEA, Vienna, 1974) p. 103

[22] H. J. Krappe, J. R. Nix, and A. J. Sierk, Phys. Rev. C 20 (1979) 992

[23] P. Möller and J. R. Nix, Nucl. Phys. A361 (1981) 117

[24] P. Möller and J. R. Nix, Atomic Data Nucl. Data Tables 26 (1981) 165

[25] J. Damgaard, H. C. Pauli, V. V. Pashkevich, and V. M. Strutinsky, Nucl. Phys. A135 (1969) 432

[26] K. T. R. Davies, A. J. Sierk, and J. R. Nix, Phys. Rev. C 13 (1976) 2385

[27] P. Möller, A. J. Sierk, T. Ichikawa, A. Iwamoto, and M. Mumpower, Phys. Rev. C 91 (2015) 024310

[28] P. Möller, R. Bengtsson, B. G. Carlsson, P. Olivius, and T. Ichikawa, Phys. Rev. Lett. 97 (2006) 162502

[29] P. Möller, R. Bengtsson, B. G. Carlsson, P. Olivius, T. Ichikawa, H. Sagawa, and A. Iwamoto Atomic Data and Nuclear Data Tables 94 (2008) 758

[30] P. Möller and J. Randrup, Phys. Rev. C 91 (2015) 044316

[31] P. Möller, A. J. Sierk, R. Bengtsson, H. Sagawa, and T. Ichikawa, Phys. Rev. Lett. 103 (2009) 212501

[32] P. Möller, A. J. Sierk, R. Bengtssson, H. Sagawa, and T. Ichikawa, Atomic Data And Nuclear Data Tables, 98 (2012) 149

[33] P. Möller, J. R. Nix, and K.-L. Kratz, Atomic Data Nucl. Data Tables 66 (1997) 131

[34] P. Möller, R. Bengtsson, K.-L. Kratz, and H. Sagawa, Proc. International Conference on Nuclear Data and Technology, April 22-27, 2007, Nice, France, (EDP Sciences, (2008) p. 69, ISBN 978-2-7598-0090-2), and http://t2.lanl.gov/nis/molleretal/publications/nd2007.html

[35] G. Audi, Midstream atomic mass evaluation, private communication (1989), with four revisions

[36] Yu. Ts. Oganessian, J. Phys. G: Nucl. Part. Phys. 34 (2007) R165

[37] P. Möller and S. G. Nilsson, Phys. Lett. 31B (1970) 283

[38] P. Möller, Nucl. Phys. A192 (1972) 529

[39] P. Möller, D. G. Madland, A. J. Sierk, and A. Iwamoto, Nature 409 (2001) 785

[40] P. Möller, A. J. Sierk, T. Ichikawa, A. Iwamoto, R. Bengtsson, H. Uhrenholt, and S. Åberg, Phys. Rev. C 79 (2009) 064304

[41] J. Randrup and P. Möller, Phys. Rev. Lett. 106 (2011) 132503

[42] J. Randrup, P. Möller, and A. J. Sierk, Phys. Rev. C 84 (2011) 034613

[43] J. Randrup and P. Möller, Phys. Rev. C 88 (2013) 064606

[44] A.N. Andreyev, J. Elseviers, M. Huyse, P. Van Duppen, S. Antalic, A. Barzakh, N. Bree, T.E. Cocolios, V.F. Comas, J. Diriken et al., Phys. Rev. Lett. 105, 252502 (2010)

[45] P. Möller and T. Ichikawa, Eur. Phys. J. A 51 (2015) 173 